Int Arch Allergy Immunol 2020;181:947-955

DOI: $10.1159 / 000509607$
Received: June 12, 2020

Accepted: June 23, 2020

Published online: August 14, 2020

\title{
CD8 Treg Cells Inhibit B-Cell Proliferation and Immunoglobulin Production
}

\author{
Sudhir Gupta Houfen Su Sudhanshu Agrawal \\ Division of Basic and Clinical Immunology, Department of Medicine, School of Medicine, University of California, \\ Irvine, CA, USA
}

\section{Keywords}

CD8 Treg · B-cell proliferation · Immunoglobulin production . ICOS - LAP- 1 - CTLA-4

\begin{abstract}
Aim: The role of CD4+ Treg in immune responses has been well established. More recently, a role of $\mathrm{CD} 8+\mathrm{T}$ regulatory cells (CD8 Treg) in the regulation of immune responses in health and autoimmune diseases has been investigated. Furthermore, different investigators have used different markers to define CD8 Treg. Finally, regulatory effects of CD8 Treg have been studied against T-cell responses; however, their role in regulating $B$-cell proliferation and immunoglobulin production has not been evaluated. Therefore, in this study we examined the effect of two types of CD8 Treg on B-cell proliferation and immunoglobulin production. Methods: Purified CD8+ T cells were activated with anti-CD3/CD28 for $48 \mathrm{~h}$ and then sorted into two different types of CD8 Treg as defined by two different sets of markers, CD8+CD183+CD197+CD45RA- and CD8+CD183+CD25 high CD278+. Purified B cells were cocultured with sorted CD8 Treg at 1:1, 1:1/2, and 1:1/4 ratios and activated with anti-CD40 and CpG. B-cell proliferation was assessed by the CFSE dye dilution assay and immunoglobulin production by the ELISA assay. Results: Our data show CD183+CD197+CD45RA-CD8 Treg significantly inhibited B-cell proliferation and inhibited IgM and IgG production but not IgA production at 1:1 ratio only. However, CD183+CD25 ${ }^{\text {high }}$ CD278+CD8 Treg inhibited significantly B-
\end{abstract}

(c) 2020 S. Karger AG, Base

www.karger.com/iaa

Karger" cell proliferation at $1: 1$ and $1: 1 / 2$ ratios and $\lg M, \lg G$, and $\lg A$ production at all ratios. Conclusion: CD8 Treg regulate B-cell responses, and $\mathrm{CD} 183+\mathrm{CD} 25^{\text {high }} \mathrm{CD} 278+\mathrm{CD} 8$ Treg are more powerful regulators of $B$-cell proliferation and immunoglobulin production than CD183+CD197+CD45RA-CD8 Treg and, therefore, may be used as preferred markers for CD8 Treg.

(c) 2020 S. Karger AG, Basel

\section{Introduction}

In humans, suppressor activity in peripheral blood mononuclear cells and $\mathrm{T}$ cells against differentiation of $\mathrm{B}$ cells to immunoglobulin-producing plasma cells was described in 1970s $[1,2]$. Following the discovery of hybridoma and development of monoclonal antibodies, Strelkauskas et al. [3] reported natural suppressor activity in OKT8+ (CD8+) T cells, whereas helper activity was in OKT4+ (CD4+) T cells. Damle and Gupta $[4,5]$ reported generation of suppressor T-cell activity in CD8+ T cells following activation with concanavalin A (Con A) and in the autologous T-T mixed lymphocyte reaction. However, the field of suppressor $\mathrm{T}$ cells came to a halt due to lack of identification of molecular basis of immune suppression.

In 1982, Damle and Gupta [6] were first to demonstrate that Con A-activated CD4+ T cells possess regulatory activity (CD4 Treg), suppressing T-cell responses

Edited by: H.-U. Simon, Bern. 
in autologous and allogeneic mixed lymphocyte reactions and response of $\mathrm{T}$ cells to pokeweed mitogen. In 1995, Sakaguchi et al. [7] further defined regulatory activity of CD4+ T cells in a subset that expressed CD25. However, CD25 was also expressed on nonregulatory CD4+ T cells, especially following activation. In 2003, they reported that FoxP3 transcription factor was responsible for mediating CD4 Treg activity [8]. Since then, FoxP3+CD4 Treg have been extensively studied in both mice and human and various diseases and disease models [9-11].

In past few years, there has been renewed interest in CD8+ T regulatory cells (CD8 Treg). Experiments in mice models of human diseases have provided strong evidence of the presence of $\mathrm{CD} 8+\mathrm{T}$ regulatory cells and their role in the control of several autoimmune disease models [1216]. Recently, human CD8+ T cells with suppressor activity have also been implicated in several autoimmune diseases [17, 18]. Shi and associates [19] have reported that human CD8+CXCR3+ (CD183+) $\mathrm{T}$ cells have the same function as murine CD8+CD122+ Treg, and suppression of CD8+ T-cell function is mediated by IL-10. These cells are CD62L+CD45RA-, similar to central memory CD8+ $\mathrm{T}$ cells. We have further characterized FoxP3+CD8 Treg as $\mathrm{CD} 8+\mathrm{CD} 183+\mathrm{CD} 25^{\text {high }} \mathrm{CD} 278+(\mathrm{ICOS}+)$. In humans, CD8+ T-cell (CD8+CD183+CD62L/CD197+CD45RA-) regulatory activity has been demonstrated against autologous and allogeneic T-cell responses; however, there are no reports of CD8 Treg activity against B-cell responses. Therefore, in this study we have examined the effect of two types of CD8 Treg (CD8+CD183+CD197+CD45RA and $\mathrm{CD} 8+\mathrm{CD} 183+\mathrm{CD} 25^{\text {high }} \mathrm{CD} 278+$, the later based upon FoxP3 expression) on B-cell proliferation and immunoglobulin production.

\section{Materials and Methods}

\section{Subjects}

Peripheral blood was obtained from 6 young healthy blood donors (age 20-50 years; 3 male and 3 female) obtained at Institute of Clinical and Translational Science, School of Medicine, University of California, Irvine. The Institutional Review Board (Human) of the University of California, Irvine, approved this study. An informed signed consent was obtained from each donor.

\section{Antibodies and Reagents}

The anti-human antibodies and isotype controls that were used included the following: directly conjugated CD25 FITC (clone-M-A251), Foxp3 Alexa647 (clone-236A/E7), CD183 (CXCR3) PE (clone-IC6/CXCR3), CD197 (CCR7) FITC (clone-150503), CD45RA APC (clone-HI100), CD45RA PE (clone-HI100), LAP-1 PerCP-Cy5.5 (clone-TW4-2F8), anti-CT-
LA-4 APC (clone-BNI3), with appropriate isotype control antibodies, and Foxp3 Buffer Set were purchased from BD Biosciences (San Jose, CA, USA). CD19 PerCP (clone-4G7-2E3), CD8 PerCP Cy 5.5 (clone-SK1), CD278 (ICOS) PerCP-Cy5.5 (cloneDX29), CD278 (ICOS) Alexa Fluor 647 (clone-DX29), and CD40/ TNFRSF5 were purchased from BD Pharmingen (San Jose, CA). EasySep Human CD19 Positive and Human CD8 Positive Selection Kits were purchased from STEMCELL Technologies (Vancouver, BC, Canada). Dynabeads Human T-Activator CD3/CD28 was purchased from Life Technologies (Grand Island, NY, USA). CpG (ODN 2006-G5) was purchased from InvivoGen (San Diego, CA, USA). CFSE was purchased from Life Technologies, Carlsbad, CA, USA. AIM V media was purchased from Life Technologies (Carlsbad, CA. USA) and FBS from Omega Scientific Inc. (Tarzana, CA, USA).

CD8+ T Cell Enrichment from PBMC and Sorting of CD8 Treg

CD8 T cells were purified from PBMC with magnetic beads using EasySep ${ }^{\mathrm{TM}}$ Human CD8 Positive Selection Kit II according to the manufacturer's protocol. The purity of CD $8+$ T cells was $>94 \%$. Enriched CD8 cells were activated with $15 \mu \mathrm{L} / \mathrm{mL}$ of Human TActivator CD3/CD28 in the RPMI medium supplemented with 5\% fetal bovine serum (FBS) for $48 \mathrm{~h}$. Cells were washed and stained with monoclonal antibodies defining two different types of CD8 Treg, CD8 PerCP cy 5.5, CD183 PE, CD197 FITC, and CD45RA APC and CD8 PerCP cy 5.5, CD183 PE, CD25FITC, and CD278 (ICOS) Alexa Fluor 647, for $20 \mathrm{~min}$. Cells were washed and sorted for CD8+/CD183+/CD25 $5^{\mathrm{High}}+/ \mathrm{CD} 278+$ and for CD8+/CD183+/ CD197+/CD45RA by using a BD FACSAria II cell sorter (Becton Dickinson, San Jose, CA, USA).

Purification of B Cells

CD19+B cells were purified from PBMC with magnetic beads using EasySepTM Human CD19 Positive Selection Kit II according to the manufacturer's protocol. The purity of $\mathrm{B}$ cells as determined by an expression of CD20 was $>93 \%$.

Labeling of B Cells with CFSE

B cells $\left(10^{7}\right.$ cells $\left./ \mathrm{mL}\right)$ were incubated at $37^{\circ} \mathrm{C}$ for 15 min with $5 \mu \mathrm{L}$ of $500-\mu \mathrm{m}$ CFSE diluted in PBS. In order to stop the reaction, $100 \mu \mathrm{L}$ of fetal calf serum (FCS) was added to per ml of cells. Cells were washed twice in RPMI-FCS and resuspended in the AIM-V medium.

Coculture for B-Cell Proliferation and Immunoglobulin Production

$B$-Cell Proliferation. CFSE-labeled B cells $\left(1 \times 10^{6}\right.$ cells $\left./ \mathrm{mL}\right)$ were cultured alone or cultured at various ratios with sorted CD8 Treg. In order to avoid any competition between CD8 Teg and B cells for culture medium in cocultures, cell numbers were accordingly adjusted in B cell alone cultures. Cells were activated with $2 \mu \mathrm{g} / \mathrm{mL}$ of anti-human CD40/TNFRSF5 antibody and $2.5 \mu \mathrm{g} / \mathrm{mL}$ of $\mathrm{CpG}$ in the AIM-V medium and incubated at $37^{\circ} \mathrm{C}$ for 5 days. CFSE-labeled B cells were stained with anti-CD19 PerCP antibody and isotype control. Ten thousand cells were acquired (FACSCalibur, Becton Dickenson, San Jose, CA, USA). In all cultures, the propidium iodide dye was used to exclude dead cells. CD19+B cells were gated and analyzed by Flow Jo software. Cell counts were performed at the end of culture with an internal bead control. 


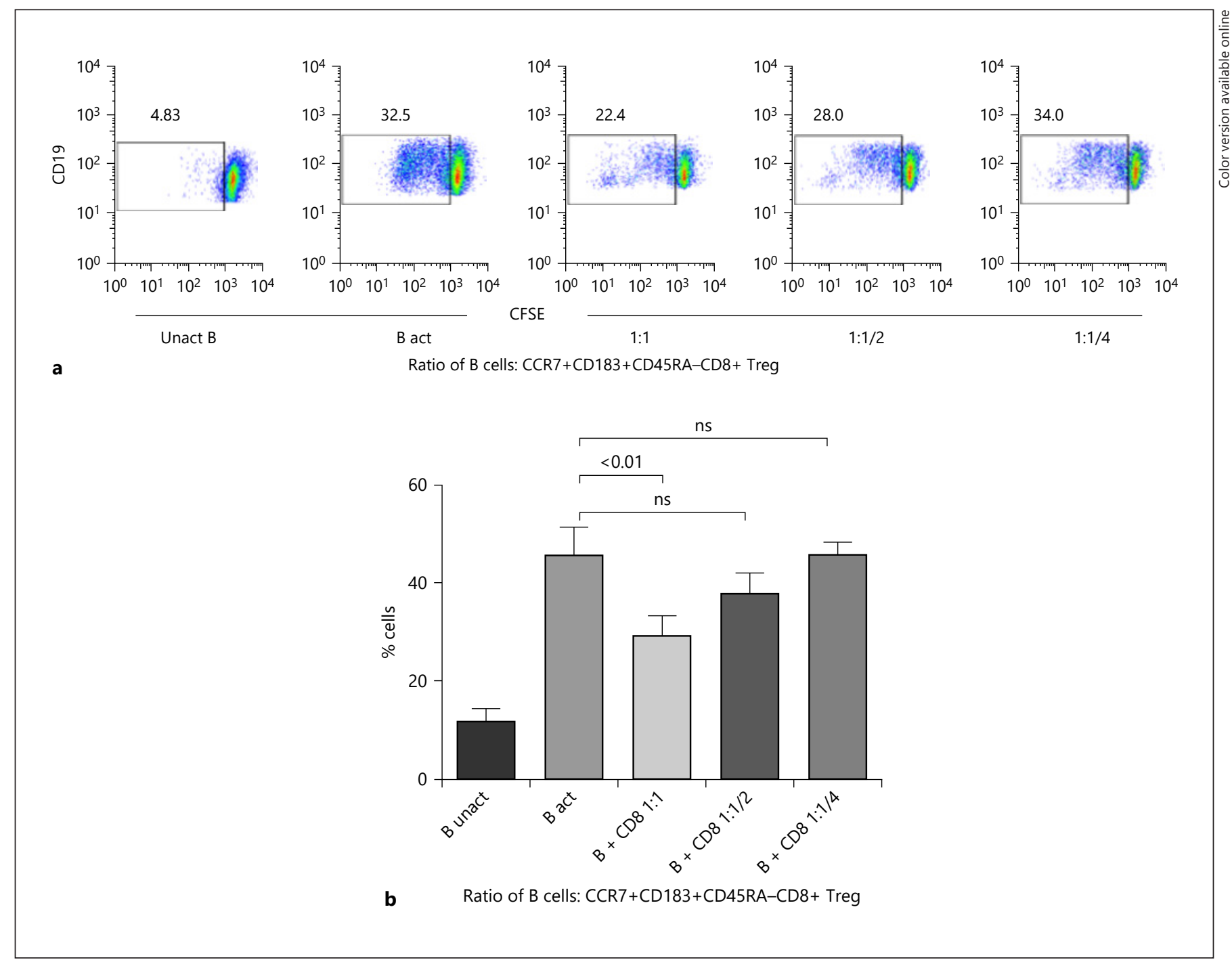

Fig. 1. Effect of CD8 Treg (CD183+CD197+CD45RA-CD8+) on B-cell proliferation. Purified CFSE-labeled B cells were cocultured in the presence or absence of various concentrations of CD183+CCR7+CD45RA-CD8 Treg and activated with anti-CD40 and CpG for 5 days. Cell division was measured by flow cytometry. a A representative flow cytometry histogram. b Cumulative data from 6 different normal donors as mean \pm SEM. Inhibition was observed only at 1:1 B:CD8 Treg ratio. CD8 Treg, CD8+ T regulatory cells.

Immunoglobulin Production. Purified B cells $\left(1 \times 10^{6}\right.$ cells $\left./ \mathrm{mL}\right)$ alone or with CD8 Treg at various ratios were activated with $2 \mu \mathrm{g} /$ $\mathrm{mL}$ of anti-human CD40/TNFRSF5 antibody and $2.5 \mu \mathrm{g} / \mathrm{mL}$ of $\mathrm{CpG}$ in the AIM-V medium and cultured at $37^{\circ} \mathrm{C}$ for 14 days. At the end of 14 days, cell viability by trypan blue dye exclusion and total cell counts were performed. Supernatants were collected and stored at $-20^{\circ} \mathrm{C}$ until assayed for detection of immunoglobulins by ELISA. Immunoglobulin levels of IgA, IgG, and IgM were determined using appropriate human ELISA kits (eBioscience/Thermo Fisher, Waltham, MA, USA) according to the manufacturer's protocol.

All experiments were performed concomitantly with both subsets of CD* Treg and under same experimental condition with the same group of subjects. Statistical analysis was performed by paired parametric Student's $t$ test using Prism 7 software. A value of $<0.05$ is considered significant.

\section{Results}

\section{CD183+CD197+CD45RA-CD8+ Treg Inhibit B-Cell} Proliferation

Purified B cells were first labeled with CFSE and then cocultured without or with sorted CD183+CD197+CD45RA- 


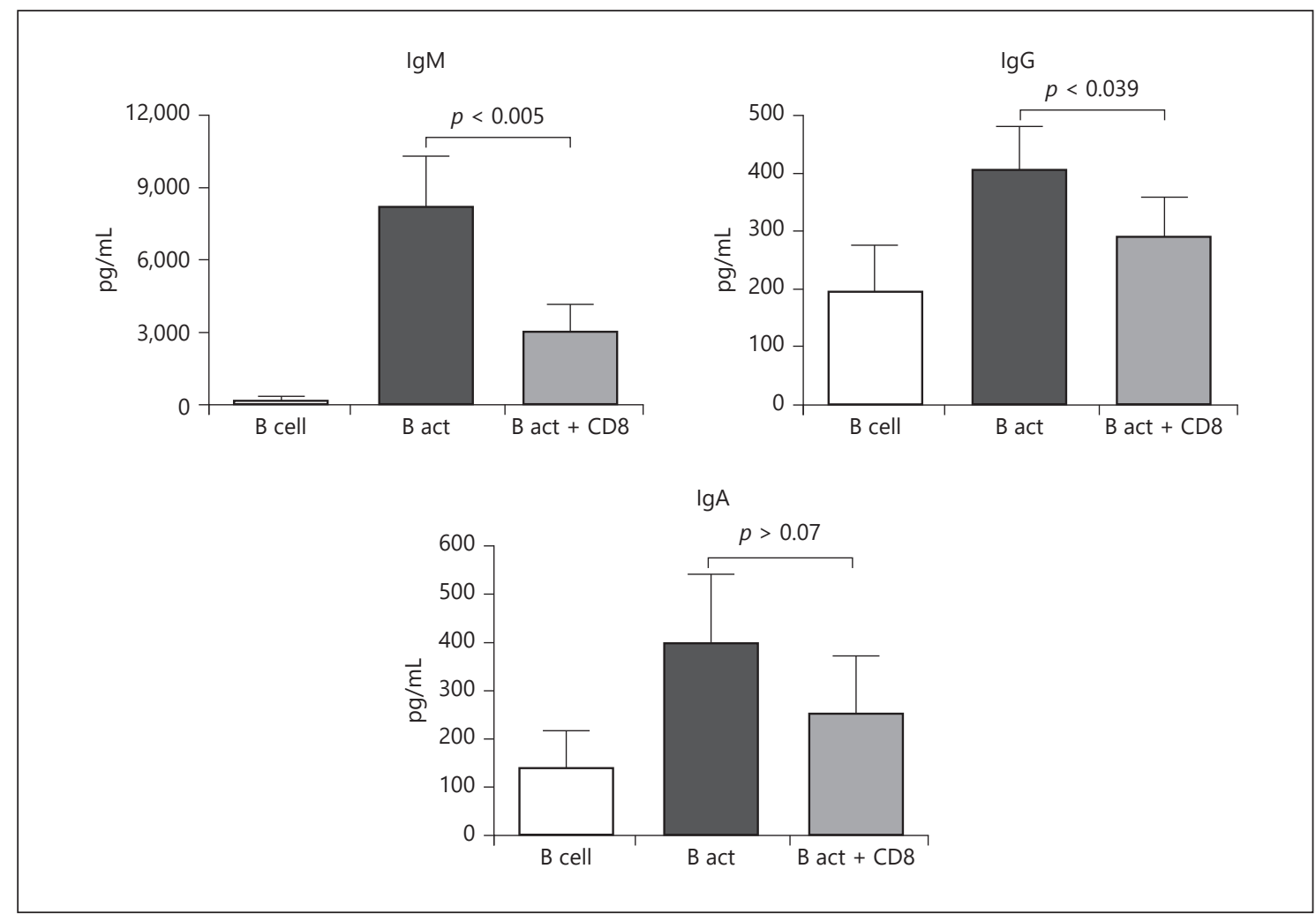

Fig. 2. Effect of CD8 Treg (CD183+CD197+CD45RA-CD8+) on immunoglobulin production. Purified B cells were cocultured in the presence or absence of CD183+CD197+CD45RA-CD8 Treg at 1:1 ratio and activated with anti-CD40 and CpG for 14 days. Supernatants were collected, and immunoglobulins were measured by the ELISA assay. Data are expressed for 6 subjects as mean \pm SEM. CD8 Treg significantly inhibited IgM $(p<0.005)$ and IgG $(p<0.039)$ production. No significant effect was observed on IgA production $(p>0.07)$. CD8 Treg, CD8+ T regulatory cells.

CD8+Treg at B:CD8 Treg ratios of 1:1, 1:1/2, and 1:1/4 and activated with anti-CD40 and CpG for 5 days, and proliferation was assayed for CFSE dilution. A representative flow cytometry histogram is shown in Figure 1a, and cumulative data from 6 different individuals are shown in Figure 1b. Data are expressed as mean \pm SEM. A significant inhibitory effect was observed only at the $\mathrm{B}: \mathrm{CD} 8 \mathrm{Treg}$ ratio of $1: 1$.

\section{CD183+CD197+CD45RA-CD8+ Treg Inhibit \\ Immunoglobulin Production}

Purified B cells were cultured alone or with CD8 Treg at various ratios, activated with $2 \mu \mathrm{g} / \mathrm{mL}$ of anti-human CD40 antibody and $2.5 \mu \mathrm{g} / \mathrm{mL}$ of $\mathrm{CpG}$, and incubated at $37^{\circ} \mathrm{C}$ for 14 days. Culture supernatants were collected, and IgM, IgG, and IgA were measured by using the ELISA kit against known standards and according to the manufacturer's protocol. Figure 2 shows data from 6 normal controls at the B:CD8 Treg ratio of 1:1. CD8 Treg significantly inhibited $\operatorname{IgM}(<0.005)$ and $\operatorname{IgG}(p<0.039)$ pro- duction. CD8 Treg tended to inhibit IgA production but without statistical significance $(p>0.078)$. No significant effect was observed on $1: 1 / 2$ and $1: 1 / 4$ ratios (data not shown).

\section{Further Characterization of CD8 Treg}

FoxP3 is a transcription factor responsible for regulatory activity. Since a number of molecules have been described to express on CD8 Treg [16, 20-22], we used several described markers on FoxP3+ and FoxP3-CD8+ $\mathrm{T}$ cells to further characterize CD8 Treg. Anti-CD3/ CD28-activated purified CD8+ T cells were stained with the following monoclonal antibodies and isotype controls: anti-CD8-PerCP-Cy5.5, anti-CD183-BV420, and anti-CD25-PE-CF595 (all from BD Bioscience, San Diego, CA, USA); anti-ICOS (CD278)-APC and anti-CTLA-4-APC (from BioLegend, San Diego, CA, USA). The cells were fixated and permeabilized using the Human FoxP3 Buffer Set from BD Pharmingen ${ }^{\mathrm{TM}}$ (San Diego, 


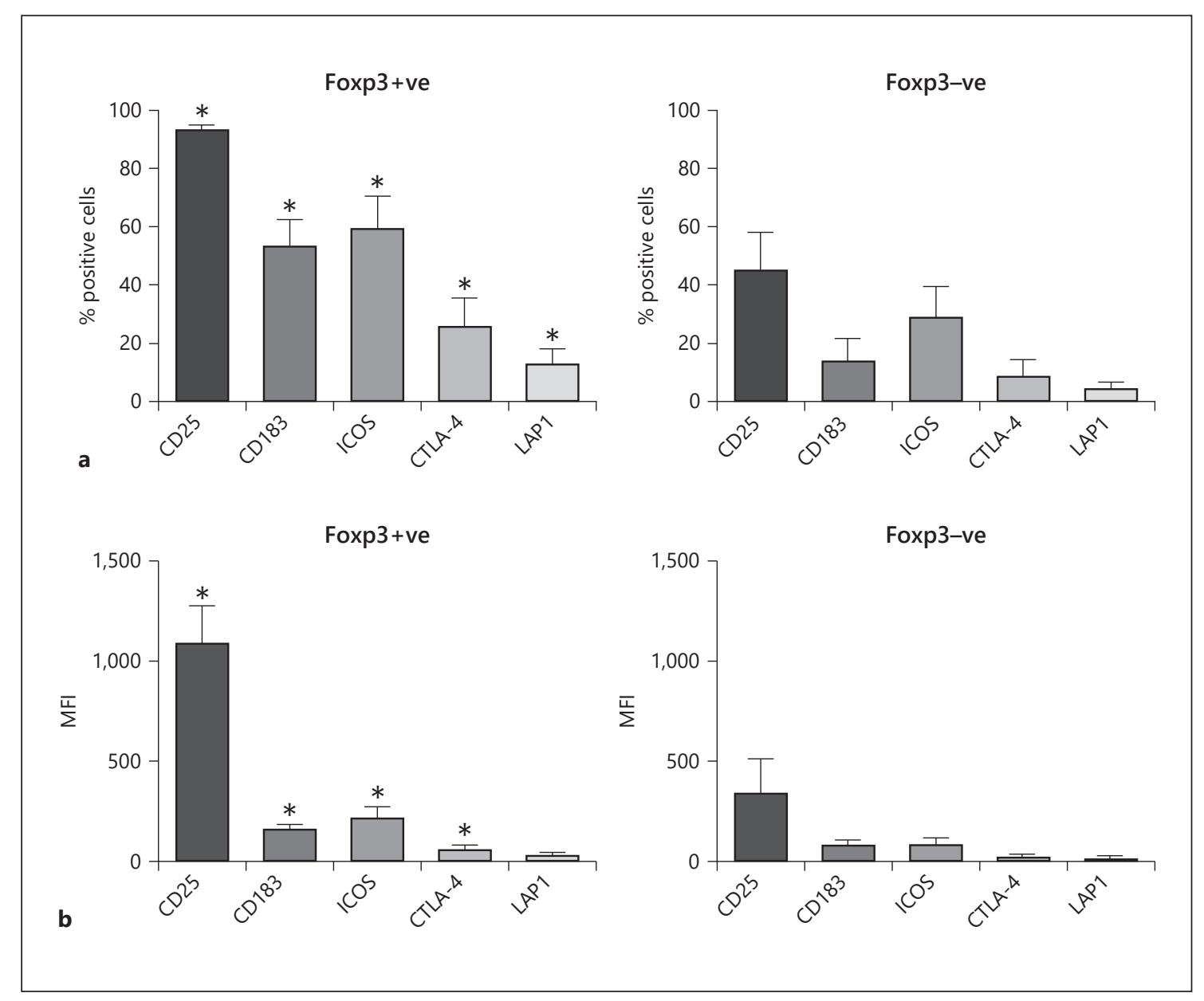

Fig. 3. Further characterization of CD8 Treg. Activated CD8+ T cells were stained with various monoclonal antibodies and isotype controls. FoxP3+ cells were gated and examined for the expression of different antigens. Expression of molecules on \% of CD8+ T cells (a) and mean fluorescence intensity (MFI) of the different molecules (b). Data are shown for 5 different subjects and expressed as mean \pm SEM. A significantly $\left(^{*}\right)$ increased $(p<0.005)$ expression of CD25, CD183, CD278, CTLA-4, and LAP-1 was observed on FoxP3+CD8+ T cells as compared with FoxP3-cells. CD8 Treg, CD8+ T regulatory cells.

CA, USA), according to the manufacturer's instructions. After wash, the cells were stained with anti-FoxP3$\mathrm{PE}$ and anti-LAP-1-PerCP-Cy5.5 for $30 \mathrm{~min}$ at room temperature. Cells were gated for FoxP3 and then examined for the expression of other markers on FoxP3+ and FoxP3-CD8+ T cells. Data in Figure 3a (\% of positive cells) and Figure $3 \mathrm{~b}$ (mean fluorescence intensity [MFI]) show that FoxP3+CD8+ T cells expressed significantly higher levels of CD25, CD278 (ICOS), CTLA-4, CD183, and LAP- 1 as compared with FoxP3-CD8 T cells. Therefore, we examined regulatory activity of sorted CD183+CD25 ${ }^{\text {high }} \mathrm{CD} 278+\mathrm{CD} 8+$ Treg on B-cell proliferation and immunoglobulin production.

\section{CD25 ${ }^{\text {high }} \mathrm{CD} 278+C D 183+C D 8+$ Treg Inhibit B-Cell Proliferation}

Purified B cells were first labeled with CFSE and then co-cultured without or with sorted $\mathrm{CD} 25^{\text {high }} \mathrm{CD} 278+$ CD183+CD8+ Treg at B:CD8 Treg ratios of 1:1, 1:1/2, and $1: 1 / 4$ and activated with $2 \mu \mathrm{g} / \mathrm{mL}$ of anti-CD40 and $2.5 \mu \mathrm{g} / \mathrm{mL}$ of $\mathrm{CpG}$ and cultured for 5 days, and proliferation was determined by the CFSE dilution assay. A representative flow cytometry histogram is shown in Figure 4a, and cumulative data from 6 different individuals are shown in Figure 4b. CD8 Treg, in a concentration-dependent manner, inhibited B-cell proliferation at B:CD8 Treg ratios of $1: 1(p<0.001), 1: 1 / 2(p<0.001)$, and $1: 1 / 4(p>$ $0.80)$. 


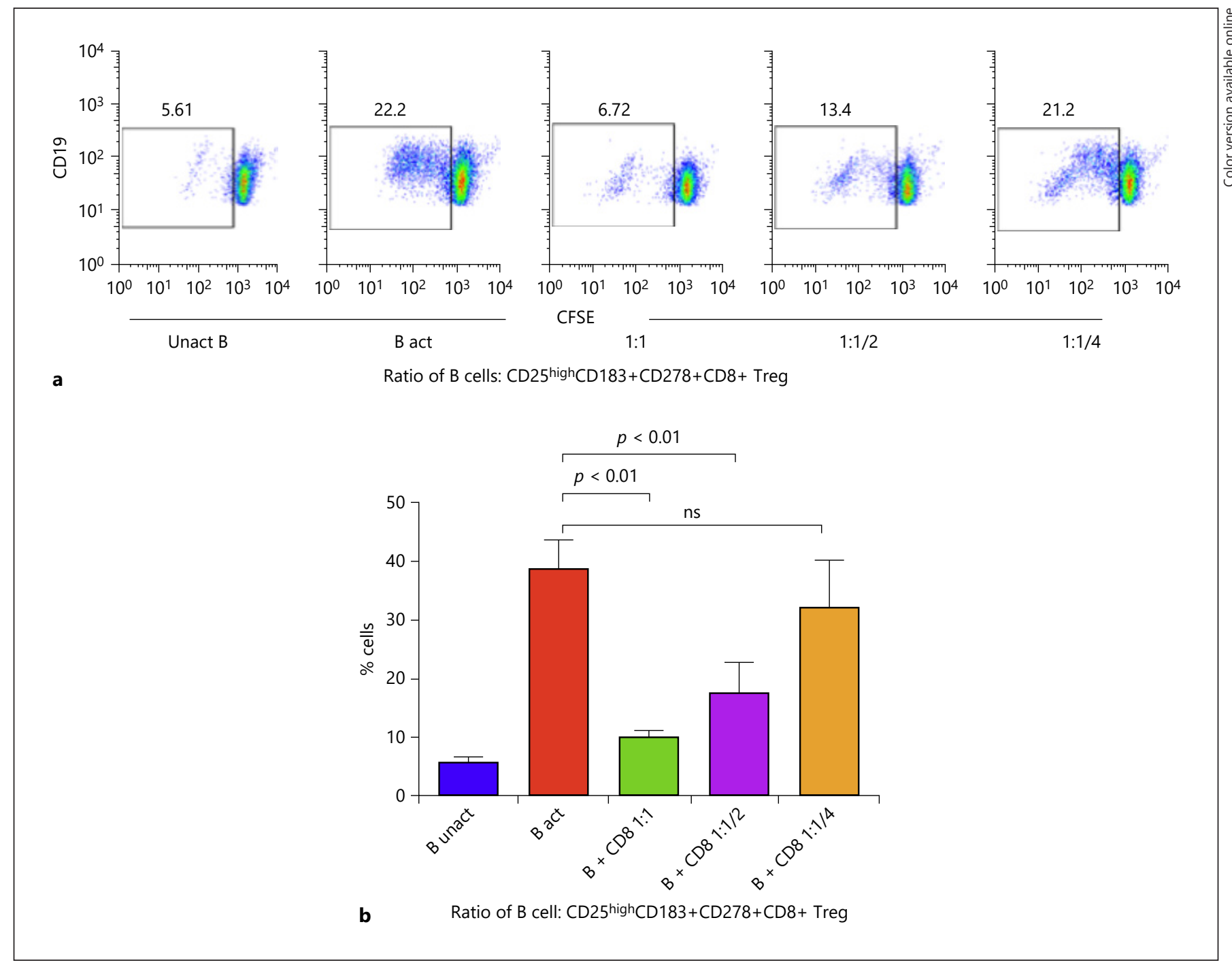

Fig. 4. Effect of CD8 Treg (CD183+CD25 high CD278+CD8+) on B-cell proliferation. Purified CFSE-labeled B cells were cocultured in the presence or absence of various concentrations of CD183+CD25 ${ }^{\text {high }} \mathrm{CD} 278+\mathrm{CD} 8$ Treg and activated with anti-CD40 and CpG for 5 days. Cell division was measured by flow cytometry. a A representative flow cytometry histogram. b Cumulative data from 6 different normal donors as mean \pm SEM. In a concentrationdependent manner, CD8 Treg inhibited B-cell proliferation. CD8 Treg, CD8+ T regulatory cells.

\section{CD25 ${ }^{\text {high }}$ CD278+CD183+CD8+ Treg Inhibit}

Immunoglobulin Production

Purified B cells were cultured alone or with CD25 ${ }^{\text {high }} \mathrm{CD} 278+\mathrm{CD} 183+\mathrm{CD} 8+$ Treg at B:CD8 Treg ratios of $1: 1,1: 1 / 2$, and $1: 1 / 4$, activated with $2 \mu \mathrm{g} / \mathrm{mL}$ of anti-human CD40 antibody and $2.5 \mu \mathrm{g} / \mathrm{mL}$ of $\mathrm{CpG}$, and incubated at $37^{\circ} \mathrm{C}$ for 14 days. Culture supernatants were collected, and IgM, IgG, and IgA were measured by using the ELISA kit against known standards and according to the manufacturer's protocol. Figure 5 shows data from 6 normal controls. CD8 Treg significantly inhibited production of all immunoglobulin isotypes at all ratios.

\section{Discussion}

Prior to the discovery of hybridoma technology to generate monoclonal antibodies, suppressor T-cell activity in humans was first shown by Waldmann et al. [1] and 

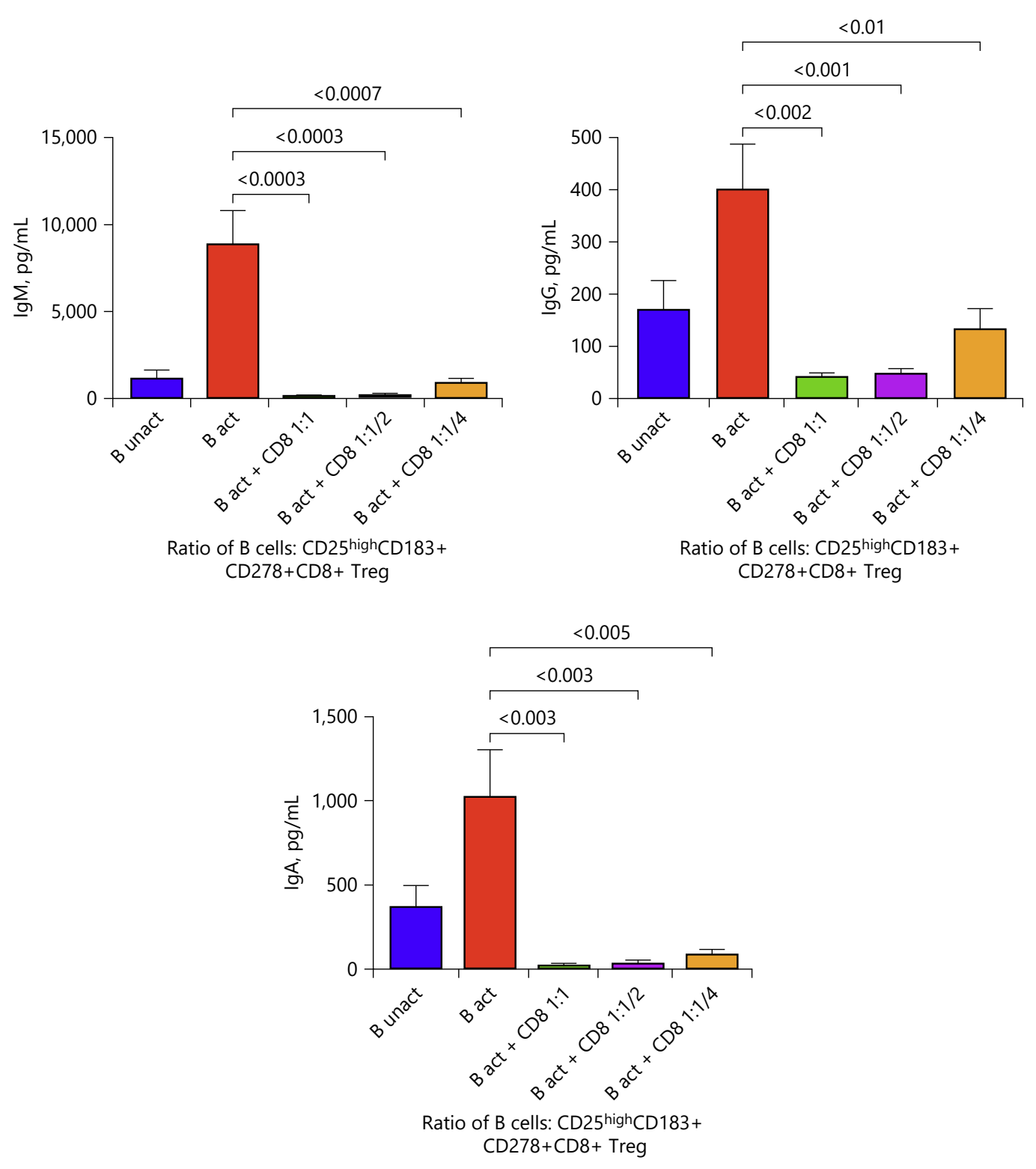

Fig. 5. Effect of CD8 Treg (CD183+CD25 $\left.5^{\text {high }} \mathrm{CD} 278+\mathrm{CD} 8+\right)$ on immunoglobulin production. Purified B cells were cocultured in the presence or absence of CD183+CD $25^{\text {high }} \mathrm{CD} 278+\mathrm{CD} 8$ Treg at various ratios and activated with anti-CD40 and CpG for 14 days. Supernatants were collected, and immunoglobulins were measured by the ELISA assay. Data are shown from 5 separate subjects (mean \pm SEM). CD8 Treg markedly inhibited IgM, IgG, and IgA production at all B:CD8 Treg ratios. CD8 Treg, CD8+ T regulatory cells.

Siegal et al. [2]. The activity of suppressor T cells was increased in patients with primary immunodeficiency and appeared to play a role in decreased immunoglobulin production by B cells. Shou et al. [23] and Gupta et al. [4] reported generation of suppressor T-cell activity follow- ing activation with Con $\mathrm{A}$, which was altered in patients with primary immunodeficiencies [24]. Schwartz et al. [25] demonstrated that Con A-activated PBMC suppressed differentiation of $B$ cells to immunoglobulin-synthesizing and -secreting plasma cells. We showed that 
Con A-activated purified T cells suppressed immunoglobulin production by $\mathrm{B}$ cells, and Con $\mathrm{A}$ activation of $\mathrm{T}$ cells was associated with increase in $\mathrm{T} \gamma$ cells that were considered suppressor T cells [4]. Following availability of monoclonal antibodies to define $\mathrm{T}$ cell subsets, Strelkauskas et al. [3] reported natural suppressor activity in OKT8+ (CD8+) T cells, whereas helper activity was in OKT4+ (CD4+) T cells. Damle and Gupta [6] and Reinherz et al. [26] reported that Con A-induced suppressor activity of $\mathrm{T}$ cells was in CD8+ T cells. Now more than 3 decades later, interest in CD8 Treg cells has revived. In a number of experimental models and human autoimmune diseases, and cancer, its role in the regulation of immune response has been established. Shi et al. [19] reported that human CD8+CD183+ T cells have the same function as murine $\mathrm{CD} 8+\mathrm{CD} 122+$ Treg, and suppression of CD8+ T-cell function is mediated by IL-10.

A number of studies have demonstrated that CD8+ Treg cells induce the expression of inhibitory receptor on monocytes and dendritic cells, suppress the proliferation of effector and memory CD4+ and T cells, and downregulate IFN- $\gamma$ production by CD8+ T cells $[22,27,28]$. However, regulatory effects of CD8 Treg on B-cell functions have not been evaluated.

Investigators have used different surface molecules including LAP-1, CD39, PD-1, and CTLA-4 to define CD8 Treg to study their functions $[16,20-22]$. In the present study, to further define CD8 Treg, we utilized all these molecules and examined their expression on FoxP3 CD8+ T cells. Our data show that FoxP3+CD8+ T cells preferentially express CD25, CD278, CD183, CTLA-4, and LAP-1. LAP-1 is an intracellular molecule. Therefore, we utilized sorted CD25 ${ }^{\text {high }} \mathrm{CD} 183+\mathrm{CD} 278+\mathrm{CD} 8+$ Treg to compare their regulatory effect from CD197+ CD183+CD45RA-CD8+ Treg cells.

In the present study, we have demonstrated that CD183+CD197+CD45RA-CD8+ Treg significantly inhibited B-cell proliferation and suppressed IgM and IgG secretion by $\mathrm{B}$ cells only at the B:CD8 Treg ratio of $1: 1$; however, they did not significantly suppress IgA production. In contrast, CD25 ${ }^{\text {high }} \mathrm{CD} 278+\mathrm{CD} 183+\mathrm{CD} 8+$ Treg significantly inhibited B-cell proliferation at B:CD8 Treg ratios of $1: 1$ and $1: 1 / 2$ and significantly suppressed all immunoglobulin isotypes, IgM, IgG, and IgA production at all B:CD8 Treg ratios. The suppression of immunoglobulin production by B cells would be consistent with inhibition of plasmablasts by CD8 Treg [29]. Furthermore, CD8 Treg, defined by both sets of markers, suppressed IgM production to a greater extent than IgG and IgA. We have reported increased CD8 Treg cells in patients with primary selective IgM deficiency [30] that may be one of the mechanisms of selective IgM deficiency.

A number of mechanisms including direct cytotoxicity, cell-to-cell contact, and via soluble factors have been reported for CD8 Treg-mediated suppression of T-cell responses. CD8 Treg have been shown to regulate T-cell responses by the production of IL-10 $[19,22]$. Chen et al. [16] reported that CD8+LAP-1+FoxP3+CD8 Treg suppress EAE that is dependent upon both TGF $\beta$ and IFN $\gamma$. We also observed significantly increased expression of LAP-1 in FOXP3+CD8 T cells as compared with FoxP3CD8 $\mathrm{T}$ cells. Several investigators have reported that cellto cell contact is required for CD8 Treg-mediated suppression [22, 31, 32]. Cosmi et al. [22] reported abrogation of CD8 Treg-mediated suppression of T-cell responses by anti-CTLA- 4 and TGF- $\beta$ antibodies. Cai et al. [32] reported CD8 Treg-mediated suppression via IL-10, TGF- $\beta$, and CTLA-4. In vivo experiments in mice have demonstrated direct cytotoxic effect of CD8 Treg [33, 34]. However in the present study, in both B-cell proliferation and immunoglobulin production assays, no significant difference was observed in the viability between B-cell-CD8 Treg cocultures and B-cell cultures alone, thereby excluding any direct cytotoxic effect of CD8 Treg responsible for decreased proliferation and immunoglobulin production. Similar to suppression of T-cell responses by CD8 Treg, inhibition of $\mathrm{B}$ cells proliferation and immunoglobulin synthesis by CD8 Treg may be mediated by both cell-tocell contact and by soluble mediators. We are currently investigating mechanisms of CD8 Treg-mediated inhibition of B-cell proliferation and Ig secretion.

In summary, CD8 Treg inhibit B-cell proliferation and differentiation into immunoglobulin-producing cells. $\mathrm{CD} 25^{\text {high }} \mathrm{CD} 278+\mathrm{CD} 183+\mathrm{CD} 8+$ Treg were more effective in regulating $\mathrm{B}$-cell responses as compared with CD183+CD197+CD45RA-CD8+ Treg. CD8 Treg-mediated suppression of antibody production may be one of the mechanisms of its role in autoimmune diseases and in antibody deficiency disorders.

\section{Statement of Ethics}

This study was approved by the Institutional Review Board (Human) of the University of California, Irvine. Each subject signed a written consent form.

\section{Conflict of Interest Statement}

The authors declare no competitive financial interests in relation to the work described in this article. 


\section{Funding Sources}

This investigation was supported by unrestricted funds from Division of Basic and Clinical Immunology, University of California, Irvine.

\section{Author Contributions}

H.S. performed majority of experiments except immunoglobulin production assay. S.A. performed immunoglobulin assay and analyzed the data. S.G. conceived the idea, designed the experiments, supervised H.S., and wrote the manuscript.

\section{References}

1 Waldmann TA, Broder S, Krakauer R, MacDermott RP, Durm M, Goldman C, et al. The role of suppressor cells in the pathogenesis of common variable hypogammaglobulinemia and the immunodeficiency associated with myeloma. Fed Proc. 1976;35:2067-72.

2 Siegal FP, Siegal M, Good RA. Suppression of B-cell differentiation by leukocytes from hypogammaglobulinemic patients. J Clin Invest. 1976;58:109-22.

3 Strelkauskas AJ, Schauf V, Wilson BS, Chess L, Schlossman SF. Isolation and characterization of naturally occurring subclasses of human peripheral blood $\mathrm{T}$ cells with regulatory functions. J Immunol. 1978;120:1278-82.

4 Gupta S, Schwartz SA, Good RA. Subpopulations of human T lymphocytes. VII. Cellular basis of concanavalin A-induced T cell-mediated suppression of immunoglobulin production by B lymphocytes from normal humans. Cell Immunol. 1979;44:242-51.

5 Damle N, Gupta S. Autologous mixed lymphocyte reaction in man. V. Functionally and phenotypically distinct human T-cell subpopulations respond to non- $\mathrm{T}$ and activated $\mathrm{T}$ cells in AMLR. Scand J Immunol. 1982;16:59-68.

6 Damle NK, Gupta S. Heterogeneity of concanavalin A-induced suppressor T cells in man defined with monoclonal antibodies. Clin Exp Immunol. 1982;48:581-8.

7 Sakaguchi S, Sakaguchi N, Asano M, Itoh M, Toda M. Immunological self tolerance maintained by activated T cells expressing IL- 2 receptor alpha chain (CD25) breakdown of a single mechanism of self tolerance causes various autoimmune diseases. J Immunol. 1995; 155:1151-64.

8 Hori S, Nomura T, Sakaguchi S. Control of regulatory T cell development by the transcription factor FoxP3. Science. 2003;299:1057-61.

9 Fontenot JD, Gavin MA, Rudensky AY. FoxP3 programs the development and function of CD4+CD25+ regulatory T cells. Nat Immunol. 2003;4:330-6.

10 Nakamura K, Kitani A, Fuss I, Pedersen A, Harada $\mathrm{N}$, Nawata $\mathrm{H}$, et al. TGF-beta 1 plays an important role in the mechanisms of CD4+CD25+ regulatory cell activity in both humans and mice. J Immunol. 2004;172:834-42.

11 Bennett CL, Christie J, Ramsdell F, Brunkow ME, Ferguson PJ, Whitesell L, et al. The immune dysregulation, polyendocrinopathy, enteropathy, X-linked syndrome (IPEX) is caused by mutations of FOXP3. Nat Genet. 2001;27:20-1.
12 Endharti AT, Okuno Y, Shi Z, Misawa N, Toyokuni S, Ito M, et al. CD8+CD122+ regulatory $\mathrm{T}$ cells (Tregs) and CD4+ Tregs cooperatively prevent and cure CD4+ cell-induced colitis. J Immunol. 2011;186:41-52.

13 Lee YH, Ishida Y, Rifa'i M, Shi Z, Isobe K, Suzuki $\mathrm{H}$. Essential role of $\mathrm{CD} 8+\mathrm{CD} 122+$ Treg in the recovery from experimental autoimmune encephalomyelitis. J Immunol. 2008;180:82532.

14 Ménager-Marcq I, Pomié C, Romagnoli P, van Meerwijk JP. CD8+CD28- regulatory T lymphocytes prevent experimental inflammatory bowel disease in mice. Gastroenterology. 2006; 131:1775-85.

15 Jiang H, Canfield SM, Gallagher MP, Jiang $\mathrm{HH}$, Jiang Y, Zheng Z, et al. HLA-E-restricted regulatory CD8+ T cells are involved in development and control of human autoimmune type 1 diabetes. J Clin Invest. 2010 Oct;120(10): 3641-50.

16 Chen M-L, Yan B-S, Kozoriz D, Weiner HL. Novel CD8+ Treg suppress EAE by TGF $\beta$ - and IFN- $\gamma$-dependent mechanisms. Eur J Immunol. 2009;39:3423-46.

17 Brimnes J, Allez M, Dotan I, Shao L, Nakazawa A, Myer L. Defect in CD8+ regulatory T cells in the lamina propria of patients with inflammatory bowel disease. J Immunol. 2005;174: 5814-22.

18 Tennakoon DK, Mehta RS, Ortega SB, Bhoj V, Racke MK, Karandikar NJ. Therapeutic induction of regulatory, cytotoxic CD8+ T cells in multiple sclerosis. J Immunol. 2006;176:11926.

19 Shi Z, Okuno Y, Rifa'I M, Endharti AT, Akane $\mathrm{K}$, Isobe $\mathrm{K}-\mathrm{I}$, et al. Human CD8+CCR3+ T cells have same function as murine CD8+CD122+ Treg. Eur J Immunology. 2009;39:2106-19.

20 Deaglio S, Dwyer KM, Gao W, Friedman D, Usheva A, Erat A, et al. Adenosine generation catabolized by CD39 and CD73 expressed on regulatory $\mathrm{T}$ cells mediate immune suppression. J Exp Med. 2007;204:1257-65.

21 Dai H, Wan N, Zhang S, Moore Y, Wan F, Dai Z. Cutting edge: programmed death-1 defines $\mathrm{CD} 8+\mathrm{CD} 122+\mathrm{T}$ cells as regulatory versus memory T cells. J Immunol. 2010;124:113043.

22 Cosmi L, Liotta F, Lazzeri E, Francalanci M, Angeli R, Mazzinghi B. et al. Human CD8+CD25+ thymocytes share phenotypic and functional features with $\mathrm{CD} 4+\mathrm{CD} 25+$ regulatory thymocytes. Blood. 2003;102: 4107-14
23 Shou L, Schwartz SA, Good RA. Suppressor cell activity after concanavalin A treatment of lymphocytes from normal donors. J Exp Med. 1976;143:1100-10.

24 Schwartz SA, Choi YS, Shou L, Good RA. Modulatory effects on immunoglobulin synthesis and secretion by lymphocytes from immunodeficient patients. J Clin Invest. 1977;59: 1176-87.

25 Schwartz SA, Shou L, Good RA, Choi YS. Suppression of immunoglobulin synthesis and secretion by peripheral blood lymphocytes by from normal donors. Proc Natl Acad Sci U S A. 1977;74:2099-103.

26 Reinherz EL, Kung PC, Goldstein G, Schlossman SF. A monoclonal antibody reactive with the human cytotoxic/suppressor T cell subset previously defined by a hetero antiserum termed TH2. J Immunol. 1980;124: $1301-7$.

27 Chang CC, Ciubotariu R, Manavalan JS, Yuan J, Colovai AI, Piazza F, et al. Tolerization of dendritic cells by TS cells: the crucial role of inhibitory receptors ILT3 and ILT4. Nat Immunol. 2002;3:237-43.

28 Long X, Cheng Q, Liang H, Zhao J, Wang J, Wang W, et al. Memory CD4+ T cells are suppressed by $\mathrm{CD} 8+$ regulatory $\mathrm{T}$ cells in vitro and in vivo. Am J Transl Res. 2017;9:63-78.

29 Gupta S, Agrawal S. In vitro effect of CD8 Treg on B cell subsets. Int Arch Allergy Immunol. 2020.

30 Louis AG, Agrawal S, Gupta S. Analysis of subsets of B cells, Breg, CD4 Treg, and CD8 Treg in adult patients with selective IgM deficiency. Am J Clin Exp Immunol. 2017;5:21-32.

31 Jarvis LB, Matyszak MK, Duggleby RC, Goodall JC, Hall FC, Gaston JS. Autoreactive human peripheral blood $\mathrm{CD} 8+\mathrm{T}$ cells with a regulatory phenotype and function. Eur J Immunol. 2005;35:2896-908.

32 Cai J, Lee J, Jankowska-Gan A, Derks R, Pool J, Mutis T, et al. Minor H antigen HA-1-specific regulator and effector CD8+ T cells and HA-1 microchimerism in allograft tolerance. J Exp Med. 2004;199:1017-23.

33 Sun D, Qin Y, Chluba J, Epplen JT, Wekerle H. Suppression of experimentally induced autoimmune encephalomyelitis by cytolytic T-T cell interactions. Nature. 1988;332:843-5.

34 Madakamutil LT, Maricic I, Sercarz E, Kumar $\mathrm{V}$. Regulatory T cells control autoimmunity in vivo by inducing apoptotic depletion of activated pathogenic lymphocytes. J Immunol. 2003; 170:2985-92. 